\title{
Otalgia secundaria o referida, lo que el clínico debe saber
}

\author{
Secondary or referred otalgia, what clinicians should know
}

\author{
Cristofer Salazar F. ${ }^{1}$, Daniela Vicencio S. ${ }^{2}$, Lara Fernández R. ${ }^{1,3}$
}

\begin{abstract}
Resumen
La otalgia es un motivo de consulta frecuente en otorrinolaringología que se puede clasificar en dos tipos: primaria y secundaria. La otalgia primaria es una consecuencia de una enfermedad otológica mientras que la secundaria o referida, surge de procesos patológicos que se originan en estructuras distintas del oído, pero que comparten vías neuronales comunes. Estas vías la componen los nervios craneales V, VII, IX y X y los nervios cervicales C2 y C3. Las causas más comunes de otalgia secundaria son la patología musculoesquelética como la disfunción de la articulación temporomandibular y el síndrome miofascial, la patología dental y las infecciones orofaríngeas. Para encontrar la etiología de la otalgia secundaria, en la mayoría de los casos, una historia clínica y examen físico acucioso resultarán suficientes, sin embargo, existe un grupo de pacientes con factores de riesgo que pudiesen requerir estudios adicionales. El objetivo de esta revisión es otorgar herramientas al lector para comprender los distintos procesos involucrados en la otalgia secundaria, conocer las patologías más relevantes relacionadas y de esta forma entregar al clínico una pauta para enfrentar este motivo de consulta. Palabras clave: Otalgia secundaria, referida, refleja, dolor de oídos.
\end{abstract}

\begin{abstract}
Otalgia is a common reason for consultation in otolaryngology and can be classified into two types: primary or secondary otalgia. Primary otalgia is a consequence of an otological disease while secondary otalgia arises from pathological processes that originate in other structures different than the ears, but share common neuronal pathways. These pathways involve cranial nerves V, VII, IX and X and cervical nerves C2 and C3. The most common causes of secondary otalgia are musculoskeletal pathology such as temporomandibular joint dysfunction and myofascial syndrome, dental pathology and oropharyngeal infections. To find the etiology of secondary otalgia, in most cases a medical history and physical examination will be sufficient, however there is a group of patients with risk factors that may require additional studies. The objective of this review is to give the reader tools to understand the different processes involved in secondary otalgia, know the most relevant related pathologies and thus give the clinician a guide to deal with this problem.
\end{abstract}

Keywords: secondary otalgia, referred otalgia, earache.
${ }^{1}$ Departamento de Otorrinolaringología, Hospital Clínico de la Universidad de Chile. Santiago, Chile. 2Escuela de Medicina, Facultad de Medicina, Universidad de Chile. Santiago, Chile. ${ }^{3}$ Clínica Santa María. Santiago, Chile.

Los autores declaran no tener conflicto de interés.

Recibido el 24 de agosto de 2019. Aceptado el 24 de marzo de 2020.

\section{Correspondencia:} Lara Fernández R. Santos Dumont 999, Independencia. Departamento de Otorrinolaringología Hospital Clínico Universidad de Chile. Santiago, Chile. Email: Ifernandezr@gmail.com

\section{Introducción}

La otalgia es un motivo de consulta frecuente en otorrinolaringología que se puede clasificar en dos tipos: otalgia primaria y secundaria $^{1,2}$. La otalgia primaria es una consecuencia de una enfermedad otológica, mientras que la otalgia secundaria, referida o refleja, surge de procesos patológicos que se originan en estructuras distintas del oído, pero que comparten vías neuronales comunes ${ }^{3,4}$.
Los orígenes de la otalgia referida (OR) pueden ser tan remotos como la cavidad craneal y el tórax, o tan malignas como ser el primer síntoma de una neoplasia de cabeza y cuello. Sin embargo, las causas más frecuentes son la patología dental, infecciones orofaríngeas, trastornos de la articulación témporomandibular y patologías de la columna cervi$\mathrm{cal}^{5}$. Para entender el origen de la OR hay que tener en cuenta que la inervación sensitiva del oído depende de una serie de nervios craneales 
y cervicales que son responsables también de la sensibilidad de otras zonas de la cabeza y el cuello. Es por ello que las patologías que surgen en las zonas inervadas por tales nervios pueden dar lugar a una $\mathrm{OR}^{1,3}$. Aunque los mecanismos son un tanto controvertidos, la teoría más aceptada es la proyección convergente, que establece que múltiples nervios convergen en una única vía neural compartida, por lo que el sistema nervioso central es incapaz de diferenciar el origen de la estimulación ${ }^{6,7}$. Dada la relevancia de este tema y lo complejo que puede ser enfrentarse a ella, el objetivo de esta revisión es otorgar herramientas al lector para comprender los distintos procesos involucrados en su desarrollo, conocer las patologías más relevantes relacionadas y, de esta forma, entregar al clínico una pauta de cómo enfrentar este motivo de consulta frecuente, que puede llegar a ser un verdadero desafío diagnóstico ${ }^{2,8}$.

\section{¿Qué tenemos que saber antes de diagnosticar una otalgia secundaria o referida?}

Para poder tener una hipótesis diagnóstica acertada, debemos conocer la epidemiología y los aspectos neuroanatómicos y fisiopatológicos de la enfermedad. De esta forma, cuando nos enfrentemos a un paciente con otalgia que al examen físico tiene un oído sano, iremos a buscar dirigidamente las posibles estructuras comprometidas, teniendo en consideración las patologías más frecuentes.

\section{Epidemiología}

La otalgia es un motivo de consulta frecuente, correspondiendo al $4 \%$ de las consultas en medicina general ${ }^{9}$. En los niños, la otalgia primaria es la más común ${ }^{7,9}$, pero en los adultos la secundaria es más frecuente, alcanzando en algunas series sobre un 50\% de las causas $s^{5,6,10,11}$. Si analizamos la otalgia por género en adultos, la otalgia primaria es más frecuente en hombres y la otalgia secundaria es más frecuente en mujeres ${ }^{7,12}$, siendo también más frecuente en este género la patología temporomandibular y las neuralgias ${ }^{6,7}$. En algunas revisiones se determina que la causa más frecuente de OR es la patología dental ${ }^{9,13}$. En la casuística de Mohammad y cols. ${ }^{12}$, esto llega a un $62,8 \%$ de los casos. Sin embargo, esta afirmación histórica se basa en evidencia controversial y poco sólida ${ }^{10}$, la cual además hay que contextualizarla en la realidad de la población estudiada, la cual en algunos casos no es extrapolable a nuestra población. La mayoría de los estudios recientes determinan que la patología cervical es la causa más frecuente de OR, seguido por la afección de la articulación temporomandibular (ATM) y en tercer lugar la patología dental ${ }^{1,4}$. Si bien los problemas de la ATM no son la causa más frecuente, esta es un motivo muy frecuente de consulta en otorrinolaringología porque aproximadamente el $65 \%$ de ellos refieren otalgia $^{1}$ y hasta un $85 \%$ de los pacientes referirán, además, plenitud ótica, vértigo, tinnitus o sensación de hipoacusia ${ }^{5}$. Por último, una causa poco frecuente de OR pero muy relevante es la patología neoplásica. Se describe que la otalgia estará presente en un $56,8 \%$ en los carcinomas nasofaríngeos, $26,0 \%$ de los cánceres de hipofaringe y un $16,7 \%$ de las lesiones malignas de la orofaringe ${ }^{5}$. Paparella describió un caso de carcinoma avanzado de esófago que se presentó únicamente con otalgia $^{6}$. Los factores de riesgo para sospechar neoplasias incluyen el consumo de alcohol y el cigarrillo, y la edad sobre 50 años ${ }^{3,6}$.

\section{Inervación del oído y vías que median el síntoma}

El oído recibe su inervación sensitiva de seis fuentes: los nervios craneales V, VII, IX y X, y los nervios espinales cervicales C2 y $\mathrm{C}^{3,5,6,9,12}$ dando la sensibilidad a las siguientes estructuras:

Nervio trigémino (V3): a través del nervio auriculotemporal recibe la información sensorial del trago, hélix, pared anterosuperior del conducto auditivo externo y la porción externa y anterior de la membrana timpánica ${ }^{2,6,8}$.

Nervio facial (VII): a través del nervio auricular posterior recibe la información sensorial de la pared posteroinferior del conducto auditivo externo y la membrana timpánica adyacente ${ }^{6,8}$.

Nervio glosofaringeo (IX): a través del nervio timpánico (nervio de Jacobson) recibe la información sensitiva de la mucosa del oído medio y tuba auditiva y la porción interna de la membrana timpánica ${ }^{2,6,8}$. 
Nervio vago $(X)$ : a través del ramo auricular del vago (nervio de Arnold), que a su vez puede recibir información del nervio facial y el glosofaríngeo, recibe la información sensitiva de la concha, la parte posterior inferior del conducto auditivo externo y segmento posterior de la membrana timpánica.

Nervios cervicales C2 y C3: a través de los nervios auricular mayor y occipital menor da la sensibilidad de la zona pre y posauricular, el lóbulo y las porciones del pabellón auricular no inervado por el auriculotemporal ${ }^{6,14}$.

De esta forma, conociendo la inervación sensitiva del oído podemos relacionar las vías nerviosas comunes que están involucradas en la otalgia refleja y las posibles estructuras anatómicas comprometidas:

Nervio trigémino: Las divisiones maxilar y mandibular del nervio trigémino reciben la inervación sensitiva de la nasofaringe, los senos paranasales, las arcadas dentarias superiores e inferiores, piso de la boca, 2/3 anteriores de la lengua y las glándulas salivales mayores. La división mandibular proporciona tanto inervación motora como sensitiva de los músculos de la masticación, el músculo tensor del tímpano y el músculo tensor del velo del paladar. Probablemente el núcleo del tracto espinal del trigémino es la vía central involucrada en generar la otalgia refleja cuando hay patologías de algunas de estas estructuras ${ }^{8}$.

Nervio facial: El nervio del conducto pterigoideo (nervio vidiano) formado por el nervio petroso superficial mayor en conjunto con fibras simpáticas que acompañan a la carótida interna, inervan la mucosa de la cavidad nasal, el seno etmoidal posterior y el seno esfenoidal. Estos estímulos llegan al ganglio geniculado del facial. El nervio intermediario puede ser el camino por el cual se desarrolla la otalgia refleja cuando hay patologías en estas estructuras ${ }^{8}$.

Nervio glosofaríngeo: El nervio glosofaríngeo recibe información sensitiva desde la nasofaringe hasta la hipofaringe. El dolor en la porción anterior de la tuba auditiva, el paladar blando, el tercio posterior de la lengua, las amígdalas palatinas y la pared lateral de la faringe, con frecuencia son referidos al oído a través del nervio timpánico ${ }^{8}$.

Nervio vago: El nervio vago transporta la información del tracto aerodigestivo inferior. La sensación de la mucosa de la vallécula, los senos piriformes y la laringe se desplaza a lo largo de la rama interna del nervio laríngeo superior. El nervio laríngeo recurrente recibe información del esófago cervical y la tráquea, además, de suministrar fibras motoras a los músculos intrínsecos de la laringe. El dolor originado en el árbol bronquial y el pulmón se refiere a través de la rama bronquial del vago. El dolor de las estructuras inervadas por el vago se refiere al oído a lo largo de la rama auricular del vago ${ }^{8}$.

\section{Patologías relevantes involucradas}

Existen diversas etiologías involucradas, de tipo inflamatorias, infecciosas, vasculares, traumáticas, tumorales o idiopáticas (Tabla $1)^{15}$. A continuación, se mencionarán las patologías más relevantes a tener en consideración, tanto por su frecuencia e impacto en la morbimortalidad, como por su relación con la otorrinolaringología.

\subsection{Sindrome de dolor miofascial}

Se caracteriza por presentar dolor difuso y sordo, de intensidad moderada, en varios sitios dentro de la región de la cabeza y el cuello, pero en particular en los músculos masticatorios asociados a bandas tensas musculares. Las otras estructuras involucradas en este síndrome son los músculos cervicales laterales y posteriores, los músculos del hombro y el hallazgo de enfermedad degenerativa de la columna cervical ${ }^{3,5,8}$. Los síntomas frecuentemente asociados son la otalgia, que a menudo es referida "en lo profundo del oído", la plenitud auditiva, la hipoacusia subjetiva (no se logra verificar en pruebas objetivas) y el tinnitus, este último descrito aproximadamente en la mitad de los $\operatorname{casos}^{3,5}$. Síntomas menos frecuentes, pero que pueden estar presentes, son el vértigo y el desequilibrio $^{3}$. El diagnóstico se realiza con la historia clínica y el examen físico palpando los distintos puntos gatillos miofasciales que desencadenarán espasmos, los cuales están en los músculos masetero, trapecio, esternocleidomastoideos y pterigoideos mediales y laterales ${ }^{3,8}$.

\subsection{Trastornos de la articulación temporo- mandibular (ATM)}

Los trastornos de la ATM son un conjunto de trastornos musculoesqueléticos que afectan 
Tabla 1. Etiología de la otalgia referida según inicio de sus síntomas ${ }^{15}$

\section{Agudo}

Eventos cardiovasculares

Infarto agudo al miocardio, aneurisma de la aorta torácica, aneurisma carotideo

\section{Arteritis de la temporal}

Edad $\geq 50$ años; cefalea de inicio reciente, claudicación mandibular, disminución de la agudeza visual que puede ser uni o bilateral, VHS elevada

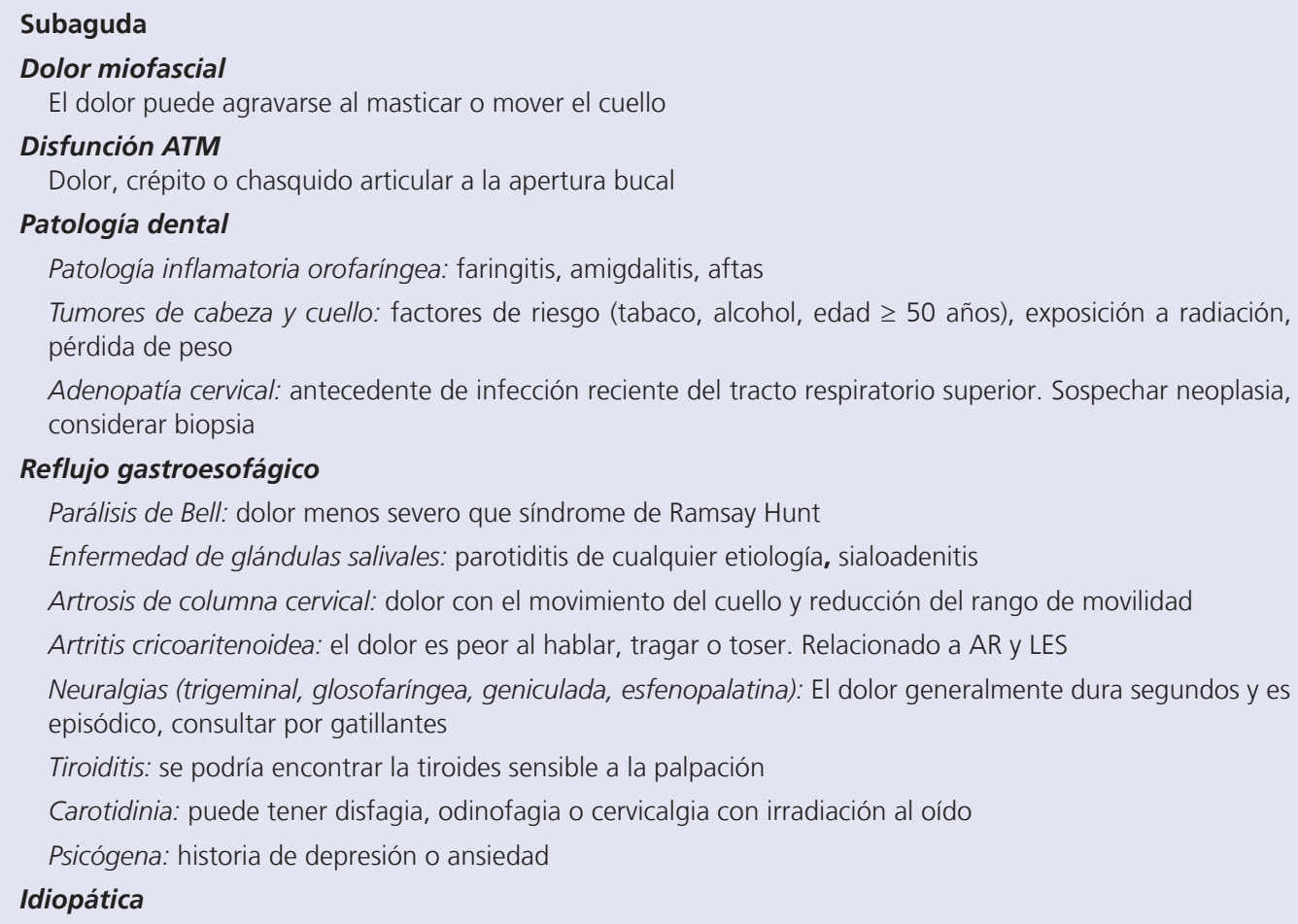

la articulación, los músculos de la masticación o ambos. El dolor puede surgir como consecuencia de una enfermedad articular intrínseca o como consecuencia de espasmos en los músculos de la masticación (temporal, masetero, pterigoideos internos y externos) ${ }^{3,5}$. El dolor miofascial se ha implicado como una causa común del componente de otalgia en los síndromes de la $\mathrm{ATM}^{1,3}$. Los trastornos de la ATM también pueden asociarse con síntomas de plenitud auditiva, tinnitus y vértigo ${ }^{9,14}$. Esto se explica por una disfunción del tensor del tímpano y del velo del paladar, ambos inervados por la rama motora del trigémino, lo que produciría un desequilibrio en el normal funcionamiento de la cadena osicular, lo que resulta a grandes rasgos en una contracción sostenida y refleja de estos músculos, lo que alteraría la presión endo y perilinfática a través de la ventana oval ${ }^{16}$.

Los hallazgos al examen físico son: crépito articular a la apertura bucal, sacudidas palpables del cóndilo a la apertura y cierre bucal y subluxación de la ATM. La presencia de crepitación en el examen no siempre implica que la ATM sea la causa de la otalgia del paciente, ya que se puede encontrar en el examen de pacientes normales ${ }^{1,3,10}$. Además, se puede detectar sensibilidad a la palpación de la ATM, en uno o más de los músculos de la masticación, y pueden estar presentes "puntos de activación" muy sensibles ${ }^{2,3}$. 


\subsection{Trastornos inflamatorios del tracto aerodigestivo}

La patología en la orofaringe, hipofaringe y la laringe son causas comunes de OR. Tenemos que destacar por su frecuencia la faringitis y amigdalitis de cualquier etiología y el dolor posamigdalectomía ${ }^{3,2,8}$. Otra patología a tener en consideración, aunque sea una causa poco probable de $\mathrm{OR}^{8}$, es la enfermedad por reflujo gastroesofágico y el reflujo faringolaríngeo, el cual puede causar otalgia por irritación del tracto aerodigestivo superior $^{5-7,14}$. La calcificación del ligamento estilohioideo que conduce a la elongación del proceso estiloides (síndrome de Eagle) es otra causa infrecuente de $\mathrm{OR}^{1,14}$, pero a tener en consideración por estar directamente relacionada con la otorrinolaringología. Consiste en dolor facial y cervical, odinofagia, globus faríngeo, disfagia y otalgia en pacientes con antecedente de amigdalectomía. El hallazgo característico es un proceso estiloides de más de $4 \mathrm{~cm}$ en la imagenología $a^{1,14}$.

\subsection{Neuralgias}

La neuralgia de los nervios trigémino, facial, glosofaríngeo y vago puede causar una variedad de dolores de oído. Estas pueden ser idiopáticas o secundarias a patologías tumorales, infecciosas (herpes) o vasculares ${ }^{8}$, por lo que toma relevancia en el estudio de la OR. Probablemente las neuralgias que más se relacionan con la otorrinolaringología son las que afectan al nervio facial en sus formas idiopática (parálisis de Bell) o asociada a herpes zoster (síndrome de Ramsay-Hunt). El dolor en la parálisis facial de Bell es menos severo y generalmente consiste en 1 a 3 días de dolor retroauricular o mastoideo que suele comenzar uno o dos días antes del inicio de la debilidad facial.

El síndrome de Ramsay-Hunt es una infección viral más grave que se manifiesta por parálisis facial, otalgia intensa y la aparición de vesículas en el conducto auditivo externo, en la membrana timpánica o alrededor de la aurícula por afección de nervios cervicales. Además, el síndrome de Ramsay-Hunt puede ir acompañado de una disfunción del VIII par, encontrando un trastorno significativo del equilibrio y una pérdida auditiva sensorioneural ${ }^{3,8}$.

\subsection{Procesos neoplásicos}

La otalgia inexplicable, especialmente en un adulto, requiere una evaluación completa de cabeza y cuello para identificar o descartar una neoplasia oculta ${ }^{3}$. Es prudente presumir que un paciente con otalgia que además tiene factores de riesgo para desarrollar neoplasias de cabeza y cuello, está cursando con una patología maligna hasta que se demuestre lo contrario ${ }^{9,14}$. La orofaringe es el sitio más frecuente de carcinoma primario del tracto aerodigestivo superior. Los tumores orofaríngeos que se presentan con otalgia incluyen carcinoma de células escamosas de la base de la lengua y de la amígdala palatina ${ }^{8}$. También los tumores en otros sitios de la cabeza y el cuello pueden presentar otalgia. Por ejemplo, los tumores de la cara anterior de la lengua pueden manifestarse como otalgia a través de la cuerda del tímpano del nervio facial. Las neoplasias malignas nasales y sinusales pueden presentar otalgia secundaria a disfunción de la tuba auditiva o afectación neural directa. En este último caso es a través de las vías aferentes de los nervios nasales posteriores superiores que van al ganglio esfenopalatino, rama de V2. Las lesiones que surgen de la fosa infratemporal pueden causar otalgia por afectación del ramo auricular del vago o del nervio timpánico ${ }^{14}$.

\subsection{Urgencias}

Si bien es poco frecuente encontrarse en este escenario, en los pacientes con diagnóstico de OR se debe descartar varias causas potenciales que pueden tener graves consecuencias si el diagnóstico se retrasa, como el infarto al miocardio (el cual se ha descrito como único síntoma la otalgia $)^{5}$, arteritis temporal, aneurisma de la aorta torácica y el aneurisma carotideo, entre otras causas aún menos frecuentes. Es por ello que se debe tener un alto índice de sospecha, sobre todo cuando se enfrenta a un paciente con factores de riesgo $0^{5,8,15}$.

\section{¿Qué tenemos que hacer para encontrar la causa de la otalgia secundaria?}

En la gran mayoría de los casos, una historia clínica y examen físico acucioso resulta suficiente para un diagnóstico preciso de la 
otalgia secundaria ${ }^{2,3}$. Sin embargo, existe un grupo de pacientes con factores de riesgo que pudiesen requerir estudio adicional ${ }^{7,9}$.

\section{Historia clínica y examen físico}

Como se ha revisado en este artículo, las posibles causas de OR son muchas (Tabla 1) ${ }^{15}$ por lo que la anamnesis tiene que seguir algunos principios que permitirán llegar al diagnóstico sin pasar por alto los factores más importantes: primero se tiene que buscar en la anamnesis los síntomas que nos orienten a las patologías más prevalentes según edad o género. Hay que tener en cuenta que alrededor de un $66 \%$ de las causas en adultos serán músculo esqueléticas (síndrome miofascial y disfunción de la $\mathrm{ATM})^{4}$. En este punto es útil preguntar por síntomas de estrés, por bruxismo o dolor de cuello recurrente ${ }^{11}$, ya que frecuentemente están relacionados con estas patologías.

Lo segundo más relevante a indagar en la historia son las patologías malignas. Si bien la mayoría de los casos de OR son secundarios a procesos benignos, la otalgia puede ser un presagio temprano de una patología subyacente grave ${ }^{6}$ por lo que se tiene que preguntar siempre por síntomas que nos orienten a malignidad, como baja de peso, disfonía crónica, disfagia progresiva, epistaxis recurrente unilateral $\mathrm{u}$ obstrucción nasal unilateral de reciente comienzo, por mencionar algunos. Indagar sobre factores de riesgo para patología maligna como el tabaquismo, consumo excesivo de alcohol o antecedentes de patologías malignas previa de cabeza y cuello o pulmonar y la exposición a radioterapia ${ }^{5}$.

Con respeto a la gravedad de los síntomas, estos no se correlacionan con la gravedad de la enfermedad, por ejemplo, las caries tienen la capacidad de producir una otalgia intensa, de carácter sordo y constante, mientras que el cáncer de laringe puede provocar una otalgia mucho menos llamativa ${ }^{6,14}$. Con respecto a la temporalidad del dolor, este nos puede orientar sobre las posibles causas. El dolor asociado a una infección generalmente es continuo (aunque puede aumentar y disminuir en intensidad) y es probable que se agrave progresivamente con el transcurso de horas o días. Es poco probable que una infección produzca un dolor que dure unas pocas horas y luego se resuelva por completo o que regrese días o semanas después por breves intervalos de tiempo. El dolor intermitente es mucho más probable que se asocie, por ejemplo, con afecciones musculoesqueléticas. La disfunción de la articulación temporomandibular y el síndrome miofascial comúnmente se presentan de esta manera ${ }^{3}$.

Lograr determinar la ubicación exacta de la otalgia puede ofrecer una pista sobre el sitio de la enfermedad. Las lesiones de la lengua anterior y el piso de la boca generalmente remiten el dolor al canal auditivo, la concha y la membrana timpánica externa a través de la división mandibular del nervio trigémino, mientras que la enfermedad de la base lateral de la lengua, la región de las amígdalas y los dos tercios inferiores de la nasofaringe envían la señal de dolor a través de nervio glosofaringaríngeo, lo que provoca un dolor intenso en el interior del oído. El nervio vago, por el contrario, inerva la supraglotis y, en estos casos, la afectación neural directa se manifiesta como un dolor superficial y menos grave de la concha y el canal auditivo. La orofaringe posterolateral y la hipofaringe producen dolor en una distribución menos predecible, debido a la inervación superpuesta de los nervios glosofaríngeo y vago ${ }^{3,5}$.

El examen físico tiene que estar dirigido en base la historia clínica, pero en general se inicia evaluando ambos oídos, deben examinarse comenzando con el oído normal o menos sintomático, incluyendo el pabellón auricular y la región pre y posauricular. Se debe anotar la evidencia de cirugía previa. El canal auditivo, la membrana timpánica y la región del ático necesitan una inspección cuidadosa. El examen otoscópico realizado por un profesional capacitado tiene una sensibilidad y una especificidad diagnósticas muy altas. El examen de la cavidad oral, la orofaringe y la nariz debe ser parte de la rutina y, en ocasiones, es necesario evaluar la hipofaringe o la laringe a través de una laringoscopía indirecta. La inspección y la palpación del cuello revelarán cualquier linfadenopatía cervical o masa. En presencia de cualquier signo o síntoma neurológico, se debe realizar un examen neurológico completo ${ }^{2,3,6,9}$. Por último, no se puede dejar de palpar la ATM, la musculatura masticatoria y cervical, buscar crépitos articulares, rangos articulares disminuidos y puntos gatillos ${ }^{15,17}$. 


\section{Exámenes complementarios}

Los exámenes complementarios están indicados cuando la anamnesis en conjunto con el examen físico no logra encontrar la causa de la otalgia $^{6,9}$, incluso buscando dirigidamente las causas más frecuentes ${ }^{1}$. La nasofibroscopía es una herramienta muy útil para evaluar la vía aerodigestiva superior que no ha podido ser evaluada con el examen físico. Nos entregará información sobre lesiones en la mucosa, aumentos de volumen, tumores, signos de reflujo faringolaríngeo, entre otros muchos hallazgos $^{14,15}$. En caso de encontrar una lesión sospechosa de malignidad en el tracto aerodigestivo superior, se debe someter al paciente a un procedimiento endoscópico con toma de biopsia para confirmar el diagnóstico clínico y ayudar en la etapificación de la enfermedad ${ }^{17}$.

Las imágenes estarían indicadas cuando se considera que un paciente tiene un alto riesgo de malignidad ${ }^{9}$, en los casos donde los síntomas de otalgia son persistentes o inexplicables $^{3}$ o hay un compromiso de los nervios craneales ${ }^{17}$. Es factible realizar una tomografía computada (TC), resonancia magnética (RM) o una combinación de ambas para la investigación de la otalgia. Con la TC se obtendrá una mejor definición ósea y del pulmón, y con la RM la evaluación de tejidos blandos será superior ${ }^{1,6}$. No se sugiere realizar imágenes de rutina en todas las otalgias secundarias, a menos que en la historia o examen físico haya indicios de necesitarlas. Así se desprende del estudio de Biggs y cols., quienes evaluaron 100 TC de pacientes con diagnóstico de OR. En $83 \%$ de los pacientes no se encontró un diagnóstico etiológico que explicara la otalgia, y, además, reportaron un 30\% de hallazgos incidentales ${ }^{1}$. Analizando estos números, el realizar de rutina una TC en la mayoría de las veces no encontraremos la causa de la OR y, además, tendremos un número importante de hallazgos incidentales que podrán requerir otro tipo de investigaciones o manejo. Por lo tanto, este tipo de imágenes pueden confundir al clínico si no se solicita con criterio.

Por último, si el paciente refiere sensación de hipoacusia, es recomendable realizar una audiometría y si tiene más de 50 años se debería solicitar un hemograma con VHS para descartar una arteritis de la temporal incipiente. En caso de existir un riesgo cardiovascular alto, solicitar un electrocardiograma, radiografía de tórax o enzimas cardíacas para descartar isquemia miocárdica o aneurisma aórtico ${ }^{18}$.

\section{Conclusión}

Como se puede desprender de esta revisión, el paciente con OR representa un reto diagnóstico para el clínico, porque al momento de encontrarnos con una otoscopia normal la gama de posibilidades diagnósticas aumenta de forma considerable, esto principalmente porque la etiología puede estar en cualquier punto dentro de los territorios inervados por los nervios craneales V, VII, IX y X y los nervios cervicales superiores $\mathrm{C} 2$ y C3. Por lo tanto, a modo de recomendación, consideramos que los pasos a seguir para hacer más fácil el estudio de la otalgia refleja son los siguientes:

1. Obtener una historia clínica completa seguida de un examen sistemático de cabeza y cuello. Esto se hace mucho más sencillo si, además, se conoce la inervación del oído y la epidemiología de la OR, porque me permitirá realizar una búsqueda mucho más dirigida.

2. Realizar una otomicroscopía para estar seguro de descartar una otalgia primaria, si con la otoscopía convencional se tienen dudas.

3. Basarse en la epidemiología para buscar inicialmente las enfermedades más frecuentes y luego ir descartando hasta llegar a las menos probables. Los primeros diagnósticos que tenemos que sospechar son la patología músculoesquelética (síndrome miofascial, disfunción de la ATM), la patología dental y faríngea.

4. En los pacientes con factores de riesgo de malignidad debe realizarse una evaluación endoscópica y eventual biopsia de las lesiones sospechosas.

5. La TC y la RM deben solicitarse cuando sospecho patología maligna, cuando hay compromiso neurológico o los síntomas son persistentes o inexplicables. Tenga cuidado en solicitar exámenes que no son necesarios porque pueden informarle un hallazgo incidental que aumente la ansiedad y costos del estudio a su paciente. En esta revisión se propone un algoritmo 


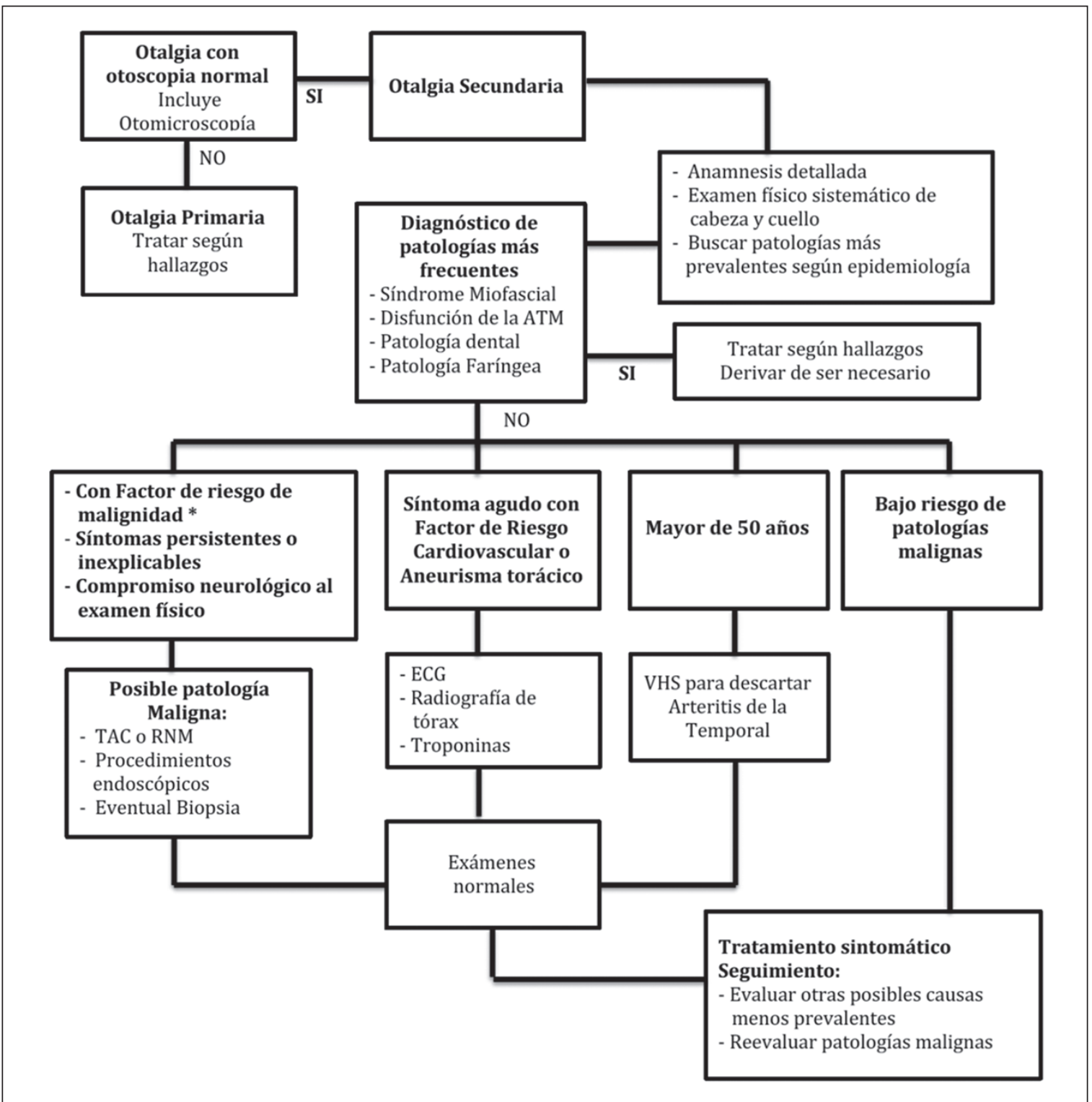

Figura 1. Algoritmo de trabajo para el enfrentamiento de la otalgia refleja. *Tabaquismo, consumo de alcohol, edad > 50 años.

de trabajo que se centra en detectar las patologías más relevantes, tanto desde el punto de vista de su frecuencia como de la morbimortalidad asociada (Figura 1$)^{7,15,17}$.

\section{Bibliografía}

1. Biggs TC, Pringle M. Computerised tomography in the investigation of otalgia of unknown origin: Our experience in 100 patients. Clin Otolaryngol.
2019;44(3):419-422. doi: 10.1111/coa.13288.

2. Majumdar S, Wu K, Bateman ND, Ray J. Diagnosis and management of otalgia in children. Arch Dis Child Educ Pract Ed. 2009;94(2):33-36. doi: 10.1136/ adc.2007.117994.

3. Neilan RE, Roland PS. Otalgia. Med Clin North Am. 2010;94(5):961-971. doi: 10.1016/j.mcna.2010.05.004.

4. Fenton JE, Uzomefuna V, O’Rourke C, Kaare M. Applying the Ts of referred otalgia to a cohort of 226 patients. Clin Otolaryngol. 2018;43(3):937-940. doi: 10.1111/coa.13069. 
5. Charlett SD, Coatesworth AP. Referred otalgia: a structured approach to diagnosis and treatment. Int $J$ Clin Pract. 2007;61(6):1015-1021. doi: 10.1111/j.17421241.2006.00932.x.

6. Chen RC, Khorsandi AS, Shatzkes DR, Holliday RA. The Radiology of Referred Otalgia. Am J Neuroradiol. 2009;30(10):1817-1823. doi: 10.3174/ajnr.A1605.

7. Kim SH, Kim TH, Byun JY, Park MS, Yeo SG. Clinical Differences in Types of Otalgia. J Audiol Otol. 2015;19(1):34-38. doi: 10.7874/jao.2015.19.1.34.

8. Weissman JL. A Pain in the Ear: The Radiology of Otalgia. http://citeseerx.ist.psu.edu/viewdoc/ download?doi=10.1.1.488.9836\&rep=rep1\&type $=$ pdf. Accessed March 15, 2019.

9. Harrison E, Cronin C. Otalgia. J Australian Family Physician. 2016;4(7):493-497.

10. Macedo J, Doi M, Oltramari-Navarro P, et al. Association between Ear Fullness, Earache, and Temporomandibular Joint Disorders in the Elderly. Int Arch Otorhinolaryngol. 2014;18(04):383-386. doi: 10.1055/s-0034-1385844.

11. Kuttila SJ, Kuttila MH, Niemi PM, Le Bell YB, Alanen PJ, Suonpää JT. Secondary Otalgia in an Adult Population. Arch Otolaryngol Neck Surg.
2001;127(4):401. doi: 10.1001/archotol.127.4.401.

12. Taziki MH, Behnampour N. A study of the etiology of referred otalgia. Iran J Otorhinolaryngol. 2012;24(69):171-176.

13. Kim DS, Cheang P, Dover S, Drake-Lee AB. Dental otalgia. J Laryngol Otol. 2007;121(12):1129-1134. doi: 10.1017/S0022215107000333.

14. Shah RK, Blevins NH. Otalgia. Otolaryngol Clin North Am. 2003;36(6):1137-1151.

15. Earwood JS, Rogers TS, Rathjen NA. Ear Pain: Diagnosing Common and Uncommon Causes. Am Fam Physician. 2018;97(1):20-27.

16. Ramírez LM, Ballesteros LE, Sandoval GP. Síntomas óticos referidos en desórdenes temporomandibulares. Relación con músculos masticatorios [Otological symptoms among patients with temporomandibular joint disorders]. Rev Med Chile 2007;135(12):15821590.

17. Visvanathan V, Kelly G. 12 minute consultation: an evidence-based management of referred otalgia. Clin Otolaryngol. 2010;35(5):409-414. doi: 10.1111/j.17494486.2010.02197.x.

18. Ely JW, Hansen MR, Clark EC. Diagnosis of ear pain. Am Fam Physician. 2008;77(5):621-628. 